\title{
Using IFN- $\gamma$ antibodies to identify the pathogens of fungal rhinosinusitis: A novel immunohistochemical approach
}

\author{
YUYAN YAN $^{1,2}$, ZUOTAO ZHAO $^{3}$, GEHONG DONG $^{1}$, YIDING HAN $^{1}$, DONGMEI YANG $^{1}$, \\ HONGYAN YIN $^{1}$, YINGSHI PIAO ${ }^{1}$, CHUNYAN HE ${ }^{1}$, CHENG TIAN $^{1}$, HONGFEI WAN ${ }^{1}$, \\ XUE LI ${ }^{1}$, YULAN JIN ${ }^{1}$, JUGAO FANG ${ }^{4}$ and HONGGANG LIU ${ }^{1,2}$ \\ ${ }^{1}$ Department of Pathology, Affiliated Beijing Tongren Hospital, Capital Medical University; \\ ${ }^{2}$ Beijing Key Laboratory of Head and Neck Molecular Diagnostic Pathology, Beijing 100730; \\ ${ }^{3}$ Department of Dermatology, First Hospital, Peking University, Beijing 100034; ${ }^{4}$ Department of ENT, \\ Affiliated Beijing Tongren Hospital, Capital Medical University, Beijing 100730, P.R. China
}

Received December 15, 2015; Accepted December 19, 2016

DOI: $10.3892 / \mathrm{mmr} .2017 .8359$

\begin{abstract}
Fungal rhinosinusitis (FRS) is commonly caused by various Aspergillus species (spp) and Mucorales fungi, and the treatment and prognosis of cases differ depending on the causative fungus. The present study describes a novel immunohistochemical method that has high sensitivity and specificity for distinguishing between these two types of fungi in patients with FRS. Three groups were included in the study. Group A included formalin-fixed paraffin-embedded blocks of 51 nasal tissue specimens of patients with FRS (27 Aspergillus spp and 24 Mucorales) that were continuously obtained from the Department of Pathology of Tongren Hospital in Beijing as the experimental group and 34 cultures (26 Aspergillus spp and 8 Mucorales) of FRS that were randomly selected from the bacterial laboratory of Tongren Hospital in Beijing to verify the staining results of the paraffin-embedded blocks. Formalin-fixed paraffin-embedded blocks of 10 esophageal cancer specimens were included in Group B as the positive control group. All specimens in Groups A and B were stained with interferon- $\gamma($ IFN- $\gamma$ ) antibody. Group C consisted of the same specimens as described in Group A, however, when performing the immunohistochemical assay, IFN- $\gamma$ antibody was replaced by PBS and this served as the negative control group. The differences in IFN- $\gamma$ immunohistochemical staining between Aspergillus spp and Mucorales were analyzed. Staining of IFN- $\gamma$ in paraffin-embedded samples was positive in $92.6 \%(25 / 27)$ of specimens in which Aspergillus spp were the causative pathogen, which was significantly
\end{abstract}

Correspondence to: Dr Honggang Liu, Department of Pathology, Affiliated Beijing Tongren Hospital, Capital Medical University, 1 Dongjiaominxiang, Dongcheng, Beijing 100730, P.R. China

E-mail: 18931107350ab@sina.com

Key words: interferon- $\gamma$, fungal rhinosinusitis, Aspergillus, Mucorales, sensitivity, specificity higher compared with specimens in which Mucorales was causative $(\mathrm{P}<0.001)$, with only $4.2 \%(1 / 24)$ of specimens staining positive for IFN- $\gamma$. Immunohistochemical staining of cell cultures was $100 \%$ positive for Aspergillus spp, whereas all Mucorales were negative. Thus, the results of the current study indicated that IFN- $\gamma$ antibody immunohistochemical staining may be used as a novel diagnostic tool to distinguish between Aspergillus spp and Mucorales when identifying the causative agent in FRS, providing a useful supplementary test to the current immunohistochemical methods in the clinical diagnosis of FRS.

\section{Introduction}

Fungal rhinosinusitis (FRS) is an infectious and/or allergic disease of the rhinosinuses caused by fungi. While considered uncommon, there have been an increasing number of cases reported over the last two decades (1). Invasive FRS (IFRS), a type of FRS, is an aggressive, often destructive and rapidly progressive infection, which is histopathologically characterized by the presence of hyphal invasion within the sinus mucosa, submucosa, blood vessels or bone and is classified as either acute or chronic (2). Acute invasive FRS (AIFRS), a subtype of IFRS, has a high mortality rate (50-80\%) in immunocompromised patients (3). Aspergillus species (spp) and Mucorales are the major pathogenic fungi implicated in FRS. Different fungi have different pathogenic mechanisms and susceptibility to different antifungal drugs, thus cases of FRS that are caused by different types of fungi vary with regard to therapeutic options and the prognosis of patients. Mucorales infection exhibits a more rapid course of progression and greater invasiveness and is more likely to invade the arterial intimal layer, which can lead to thrombosis, hemorrhagic and ischemic necrosis and is, therefore, associated with high mortality rates $(4,5)$. Currently, amphotericin B is the primary drug used to treat patients infected with Mucorales and intravenous voriconazole is the primary drug for Aspergillus spp infection. Accurate and timely diagnosis of the fungal species is of clinical importance; however, sensitive diagnostic assays have yet to be developed. 
At present, there are various laboratory tests that are used to identify fungi types, including culture, serological differentiation, molecular techniques and histopathological analysis (6). Because of its simplicity, the existence of established methods, reasonable cost and relatively fast and accurate diagnostic performance, histopathological analysis is one of the major methods applied to identify causative fungi clinically. Histopathological analysis includes the following staining techniques: Hematoxylin and eosin (H\&E), Periodic Acid-Schiff (PAS), Gomori methenamine silver (GMS) and mucin 5B (MUC5B) immunohistochemical staining. Of the staining methods mentioned, MUC5B immunohistochemical staining has strong specificity and high sensitivity as it is based on an antigen-antibody response. However, not all Aspergillus spp cases are positively identified with MUC5B immunohistochemical staining. Additional immunohistochemical staining methods with strong specificity and high sensitivity are required to improve the histopathological diagnosis of FRS.

The present study aimed to develop a novel immunohistochemical staining assay to differentiate between Aspergillus spp and Mucorales, the two major types of pathogenic fungi implicated in FRS. Interferon- $\gamma(\mathrm{IFN}-\gamma)$ is a protein dimer that has an essential role in the innate and adaptive phases of an immune response $(7,8)$. Although protein is a major component of the fungal cell wall, the presence of the IFN- $\gamma$ antigen on the fungal cell wall has not yet been demonstrated. AIFRS animal pre-experiments revealed that Aspergillus fumigatus stained positive for IFN $-\gamma$. The hyphae and conidia of A.fumigatus were stained diffuse brown on the cell wall (Fig. 1). Therefore, it was hypothesized that the IFN- $\gamma$ antigen may be specific to Aspergillus spp and that IFN- $\gamma$ antibody may be used as a diagnostic marker to differentiate between Aspergillus spp and other major pathogenic fungi that cause FRS, including Mucorales. Validation of these assumptions may provide a novel method for identifying the causative fungal type in FRS with high specificity and sensitivity, which may allow earlier diagnosis and appropriate treatment of FRS. In the current study, an IFN- $\gamma$ antibody immunohistochemical assay was performed on formalin-fixed paraffin-embedded nasal tissue specimens of FRS patients and H\&E, PAS and GMS staining was performed for comparison, to observe the differences in the staining patterns of Aspergillus spp and Mucorales. In addition, IFN- $\gamma$ antibody was used to stain cultures of Aspergillus spp and Mucorales, derived from FRS patients, to validate the results of the immunohistochemical assay on formalin-fixed paraffin-embedded specimens of FRS.

\section{Materials and methods}

Samples. Formalin-fixed paraffin-embedded blocks of 51 nasal tissue specimens of FRS patients (30 males and 21 females, mean age 32 years old; 27 infected by Aspergillus spp and 24 infected by Mucorales (Rhizopus sp.) were obtained from the Department of Pathology of Beijing Tongren Hospital. Samples were randomly selected based on a diagnosis of FRS from pathological and clinical data and fungal culture results between 2005-2012, which was when the FRS diagnosis was determined. Samples that were missing any of the aforementioned data were excluded. The cases in the present study included IFRS, fungal balls and allergic FRS. The types of causative fungi were blindly determined by two experienced pathologists following assessment of pathological sections stained with H\&E, PAS and GMS, combined with clinical features and cultures, before immunohistochemistry assays were performed. The present study was approved by the ethics committee of the Beijing Tongren Hospital (Beijing, China).

Formalin-fixed paraffin-embedded blocks of 10 esophageal cancer specimens isolated from patients were randomly selected from the Department of Pathology of Beijing Tongren Hospital and blindly determined as such by two experienced pathologists. Cultures were provided by the bacterial laboratory of Tongren Hospital in Beijing. Randomly selected Aspergillus spp (A. fumigatus, $\mathrm{n}=10 ;$ A. flavus, $\mathrm{n}=8$; and A. terreus, $\mathrm{n}=8$ ) and Mucorales cultures $(n=8)$ were cultured for 5 days at $25^{\circ} \mathrm{C}$ on Vogel's glucose agar.

Groups. Group A was the experimental group and consisted of formalin-fixed paraffin-embedded blocks of 51 FRS specimens (27 Aspergillus spp and 24 Mucorales) and 34 cultures of these specimens (26 Aspergillus spp and 8 Mucorales). Group $B$ consisted of formalin-fixed paraffin-embedded blocks of 10 esophageal cancer specimens, which served as the positive control group. Group C was the negative control group, in this group, IFN- $\gamma$ antibody was replaced by PBS when performing immunohistochemical assay of Group A specimens.

Immunohistochemical assay of formalin-fixed paraffin-embedded specimens. Sections of formalin-fixed paraffin-embedded specimens $(3 \mu \mathrm{m})$ were dried in an oven at $37^{\circ} \mathrm{C}$ for $30 \mathrm{~min}$, deparaffinized three times for $5 \mathrm{~min}$ in xylene and rehydrated with graded ethanol solutions and distilled water. Endogenous peroxidase in the sections was deactivated by incubating in $3 \% \mathrm{H}_{2} \mathrm{O}_{2}$ for $10 \mathrm{~min}$. Following rinsing with distilled water, antigen retrieval was performed using EDTA solution at a high temperature $(800 \mathrm{~W})$ in a microwave oven for $8 \mathrm{~min}$. After cooling in distilled water for $5 \mathrm{~min}$ and rinsing with PBS twice for $5 \mathrm{~min}$, sections were subsequently incubated with primary antibody (IFN- $\gamma$ antibody; orb10878; 1:200; Biorbyt Ltd., Cambridge, UK) overnight at $4^{\circ} \mathrm{C}$. The sections were subsequently rinsed with distilled water and PBS, and EnVision+ horseradish peroxidase rabbit antibody was added (K5007; Dako; Agilent Technologies, Inc., Santa Clara, CA, USA) for $30 \mathrm{~min}$ at room temperature $\left(25^{\circ} \mathrm{C}\right)$. Following washing with distilled water and PBS, the sections were incubated in 3,3'-diaminobenzidine solution (Dako; Agilent Technologies, Inc.) for 2-3 min. Chromogenic time was determined by observation using a light microscope. The sections were washed in distilled water, counterstained with hematoxylin and dehydrated with xylene prior to mounting. Esophageal cancer specimens were analyzed as positive controls using the same method. In negative controls, primary antibody was replaced by PBS.

Immunocytochemistry of cultures. Mucor cultures were added to slides, fixed in $95 \%$ ethanol for $\sim 1 \mathrm{~h}$ at room temperature and incubated with the aforementioned IFN- $\gamma$ antibody (1:200; Biorbyt) overnight at $4^{\circ} \mathrm{C}$. The remaining procedures were the same as those described in the previous 
Table I. IFN- $\gamma$ expression in Aspergillus spp and Mucorales formalin-fixed paraffin-embedded specimens.

IFN- $\gamma$ expression

\begin{tabular}{lcc}
\cline { 2 - 3 } Fungal type & Positive cases (\%) & Negative cases (\%) \\
\hline Aspergillus spp $^{\mathrm{a}}$ & $25(92.5)$ & $2(7.4)$ \\
Mucorales $^{\mathrm{b}}$ & $1(4.2)$ & $23(95.8)$ \\
\hline
\end{tabular}

${ }^{\mathrm{a}} \mathrm{P}<0.001$ vs. Aspergillus spp group; ${ }^{\mathrm{b}} \mathrm{P}<0.001$ vs. Mucor group. Total cases, $\mathrm{n}=51$; Aspergillus spp cases, $\mathrm{n}=27$; Mucorales cases, $\mathrm{n}=24$. IFN- $\gamma$, interferon- $\gamma$; spp, species.

section for the immunohistochemical assay of formalin-fixed paraffin-embedded specimens. Esophageal cancer specimens were used as positive controls. PBS was used instead of primary antibody in the negative controls. The immunohistochemical processes described were also repeated with another IFN- $\gamma$ antibody (PTG:15365-1-AP; 1:100; Proteintech Group, Inc., Chicago, IL, USA) to verify the results.

Statistical analysis. Statistical analyses were performed using SPSS version 17.0 (SPSS, Inc., Chicago, IL, USA). The significance of the results was evaluated by the $\chi^{2}$ test. $\mathrm{P}<0.05$ was considered to indicate a statistically significant difference.

\section{Results}

The majority of Aspergillus spp have segmented forms and a shape that resemble antlers. Mucorales species are thicker and the majority have right angled hyphae. However, in certain clinical cases, Aspergillus spp become thicker and deformed, perhaps affected by the clinical characteristics of patients or the process of preparing sections, making it difficult to distinguish them from other fungi based on simple morphology. Thus, a highly specific immunohistochemical method to identify fungal types is required. The standard for a positive immunohistochemistry result in the present study was a brown staining of the cell wall or cytoplasm of the fungus.

Immunohistochemistry of formalin-fixed paraffin-embedded specimens. The results of immunohistochemical staining with IFN- $\gamma$ antibody in formalin-fixed paraffin-embedded FRS specimens are presented in Table I and Fig. 2. IFN- $\gamma$ expression was positive in 25 and negative in 2 Aspergillus spp specimens. Therefore, the IFN- $\gamma$-positive rate in Aspergillus spp was $92.6 \%$ (25/27). The hyphae and conidia of Aspergillus spp exhibited diffuse brown staining on the cell wall (Figs. 3, 4 and 5). The two negative Aspergillus cases were fungal balls. Aspergillus spp specimens from cases with allergic FRS and IFRS were all stained positive. By contrast, IFN- $\gamma$ expression was negative in all Mucorales cell walls (Fig. 6), with the exception of one case, therefore, IFN- $\gamma$ staining was positive in only $4.2 \%$ of Mucorales specimens; this was significantly lower than the percentage of Aspergillus spp specimens that stained positive $(\mathrm{P}<0.001)$.

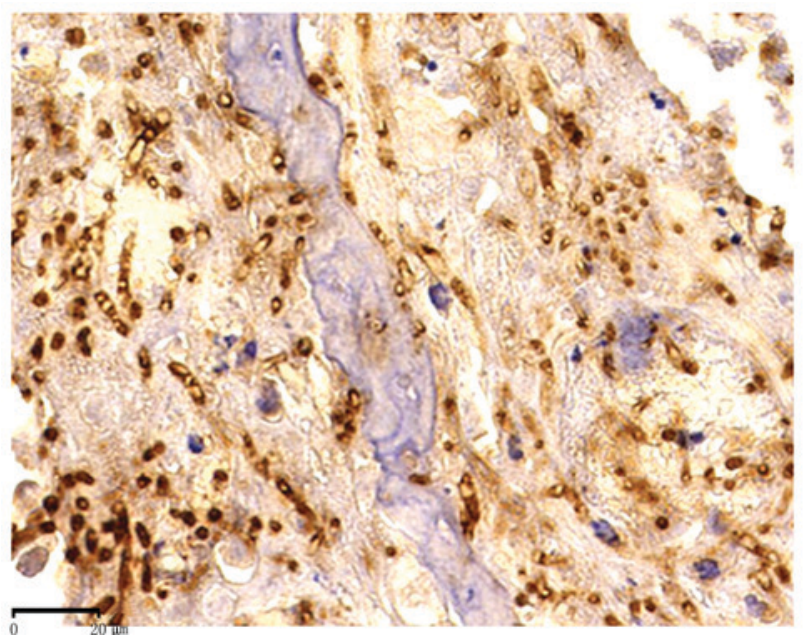

Figure 1. IFN- $\gamma$ immunostaining of Aspergillus fumigatus in an acute invasive fungal rhinosinusitis rat model in the pre-experiment. The cell walls of the hyphae are positively stained for IFN $-\gamma$ (x40 magnification). IFN- $\gamma$, interferon- $\gamma$.

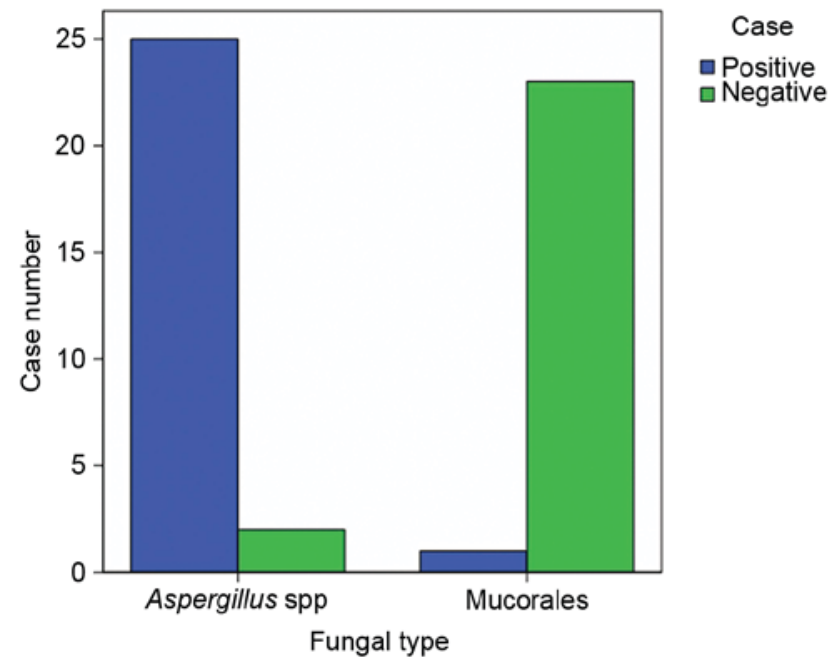

Figure 2. Number of cases that exhibited positive and negative interferon- $\gamma$ expression in Aspergillus spp and Mucorales formalin-fixed paraffin-embedded specimens.

Immunocytochemistry of cultures. The results of immunocytochemical staining of cultures for IFN- $\gamma$ are summarized in Table II, which demonstrates that positive immunoreactivity was observed in all A. fumigatus (Fig. 7), A. flavus (Fig. 8) and A.terreus (Fig. 9) cultures. The positive staining rate for Aspergillus spp was $100 \%$ (26/26), whereas all Mucor spp (Fig. 10) stained negative for IFN- $\gamma$. The cell walls of positive hyphae and conidia exhibited diffuse brown staining. A significant difference in IFN- $\gamma$ expression was observed between Aspergillus spp and Mucor spp $(\mathrm{P}<0.001)$. In the positive control group (Group B), all cancer cell membranes exhibited positive brown staining, while all specimens in the negative control group (Group C) exhibited negative staining results. Identical immunohistochemical staining results from the paraffin-embedded sections and the cultures were obtained with the two IFN- $\gamma$ antibodies from different manufacturers. 


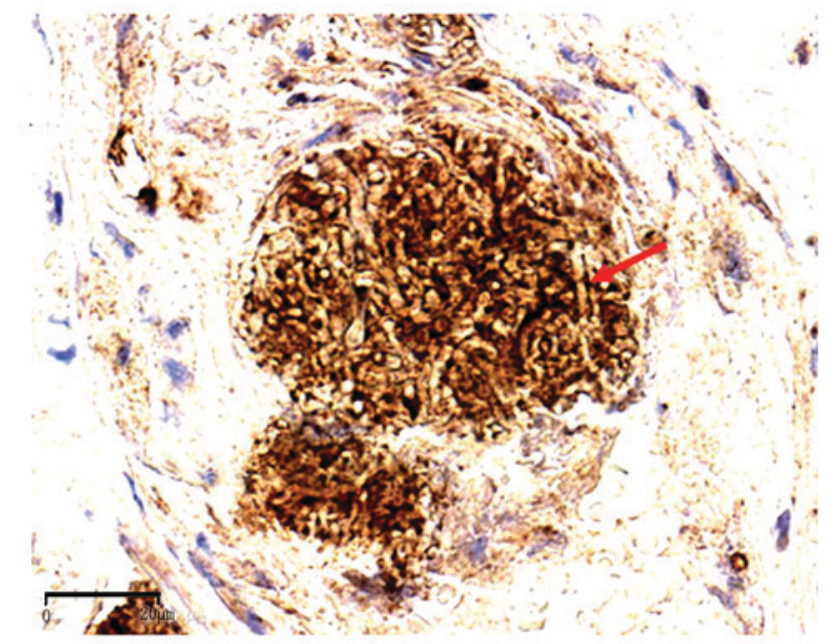

Figure 3. IFN- $\gamma$ immunostaining of Aspergillus flavus invading the vessels of human rhinosinuses. The cell walls of the hyphae are positively stained for IFN- $\gamma$ (x40 magnification). IFN- $\gamma$, interferon- $\gamma$.

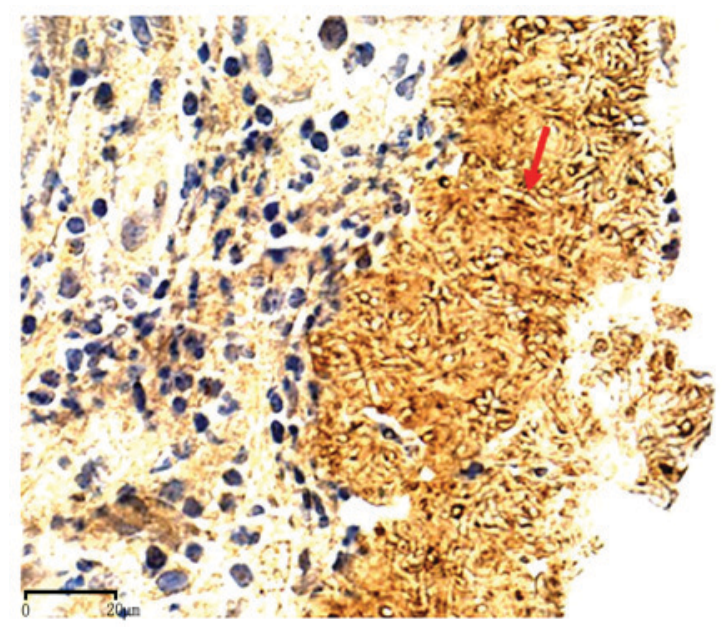

Figure 4. IFN- $\gamma$ immunostaining of Aspergillus fumigatus in a fungal ball of rhinosinus. The cell walls of the hyphae are positively for IFN- $\gamma$. x40 magnification. IFN- $\gamma$, interferon- $\gamma$.

\section{Discussion}

The present study performed immunohistochemical staining for IFN- $\gamma$ in samples of FRS and may have developed a novel diagnostic immunohistochemical approach to distinguish between types of fungi that are associated with FRS, which may increase the potential for earlier and more accurate diagnosis and treatment of FRS.

FRS was once considered to be a rare disorder; however, it is currently reported with increasing frequency worldwide (9-11). In the current classification system, FRS is classified into invasive and noninvasive types (12). Based on guidelines from the International Society for Human and Mycology Group, IFRS may be classified as acute, chronic or granulomatous, while the non-invasive types of FRS are allergic FRS and fungus balls (13). IFRS is a lethal disease that has a fatality rate of $50-80 \%$ in AIFRS (3). Currently, surgery and antifungal drugs are the major treatments for FRS. Early, accurate diagnosis and systematic antifungal

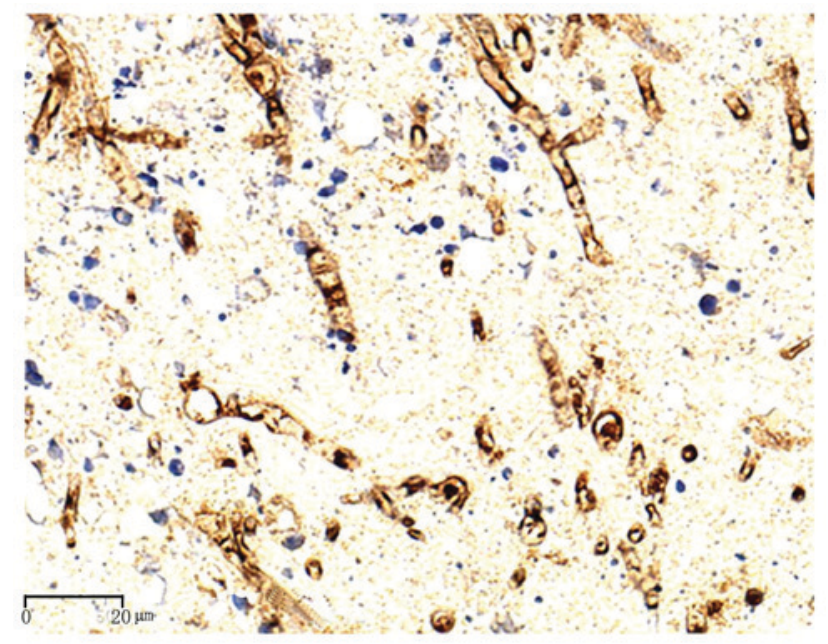

Figure 5. IFN- $\gamma$ immunostaining of Aspergillus fumigatus in allergic fungal rinosinusitis. The cell walls of the hyphae are positively stained for IFN- $\gamma$ (x40 magnification). IFN- $\gamma$, interferon- $\gamma$.

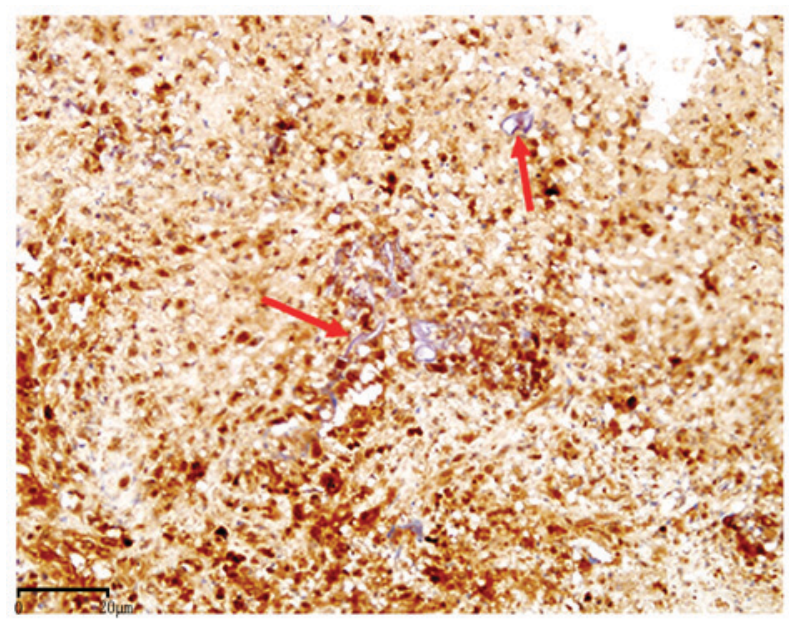

Figure 6. IFN- $\gamma$ immunostaining of Rhizopus (a common genus of Mucorales) in invasive fungal rhinosinusitis, which was negative for IFN- $\gamma$ (x40 magnification). IFN- $\gamma$, interferon- $\gamma$.

drug treatment is critical for the management of FRS. Aspergillus spp and Mucorales are the major types of pathogenic fungi that cause FRS. Aspergillus spp account for $80 \%$ of FRS cases, while Mucorales are the most aggressive and dangerous pathogens that have been implicated in FRS, which means they are associated with a high mortality rate $(3,14,15)$. The pathogenic mechanism of different fungi can vary and they may also be susceptible to different antifungal drugs. Therefore, a method that can rapidly and accurately identify the types of fungi that are causative in different FRS cases is key for early diagnosis and the appropriate treatment of FRS.

In recent years, various methods have been developed to identify the types of fungi that are implicated in FRS cases. Culture and histopathology-based tests have been supplemented with molecular and proteomic techniques, and also antigen detection methods (16-18). However, all methods possess limitations. The culture method is insensitive, has potential for laboratory contamination and often takes too 
Table II. IFN- $\gamma$ expression in of Aspergillus spp and Mucor spp cultures.

IFN- $\gamma$ expression

Fungal type

Positive cases (\%) Negative cases (\%)

\begin{tabular}{lcl}
\hline Aspergillus spp $^{\mathrm{a}}$ & $26(100)$ & $0(0)$ \\
Mucor $^{\mathrm{b}}$ & $0(0)$ & $8(100)$
\end{tabular}

${ }^{\mathrm{a}} \mathrm{P}<0.001$ vs. Aspergillus spp group; ${ }^{\mathrm{b}} \mathrm{P}<0.001$ vs. Mucor group. Total cases, $\mathrm{n}=34$; Aspergillus spp cases, $\mathrm{n}=26$; Mucor cases, $\mathrm{n}=8$. IFN- $\gamma$, interferon- $\gamma$; spp, species.

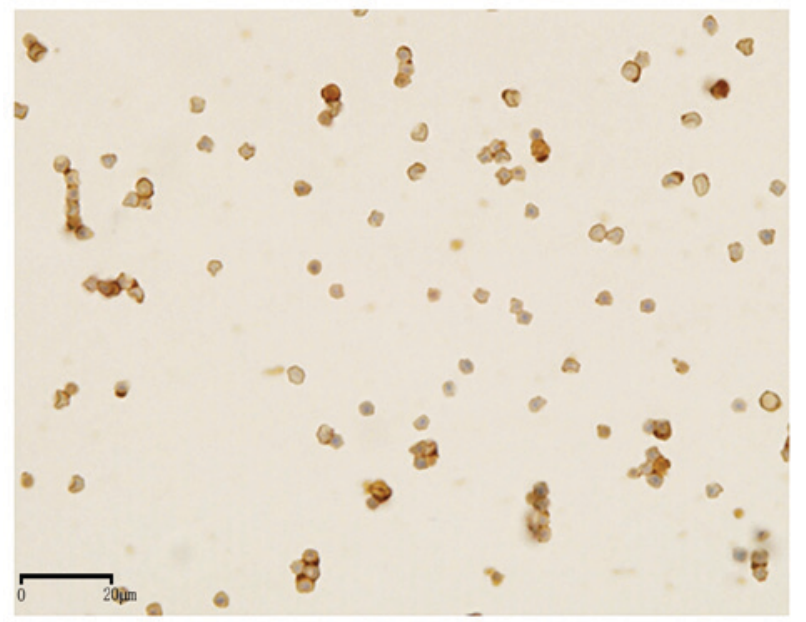

Figure 7. IFN- $\gamma$ immunostaining of Aspergillus fumigatus cultures. The cell walls of conidia are positively stained for IFN- $\gamma$ (x100 magnification). IFN- $\gamma$, interferon- $\gamma$.

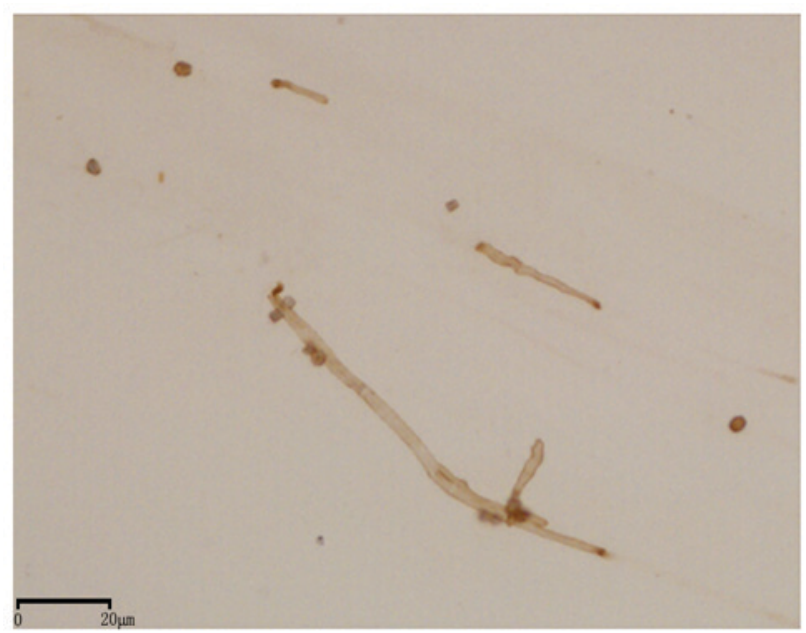

Figure 8. IFN- $\gamma$ immunostaining of Aspergillus flavus cultures. The cell walls of hyphae and conidia are positively stained for IFN $-\gamma$ ( $x 40$ magnification). IFN- $\gamma$, interferon- $\gamma$.

long (usually 5 to 12 days) to obtain results. Although molecular assays, including nucleic acid amplification tests and nucleic acid hybridization, have potential as they exhibit high sensitivity and specificity, the lack of test standardization

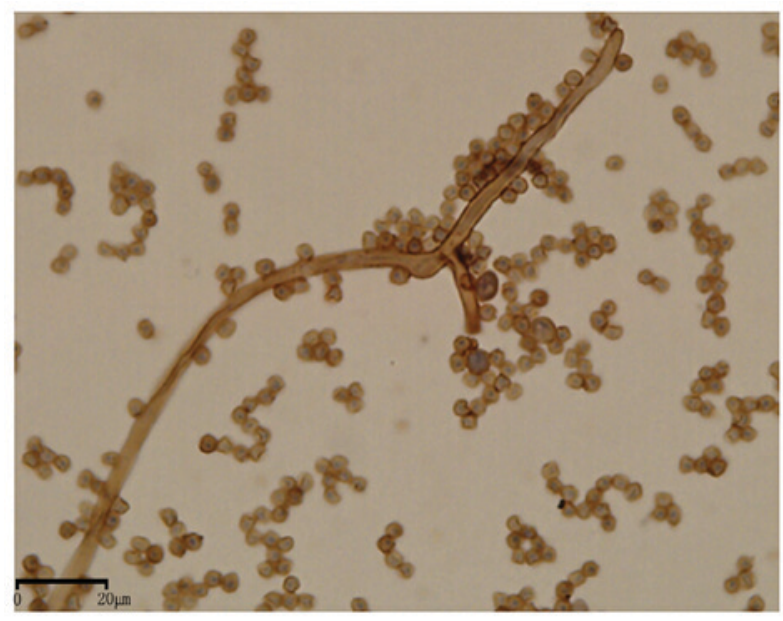

Figure 9. IFN- $\gamma$ immunostaining of Aspergillus terreus cultures. The cell walls of hyphae and conidia are positively stained for IFN- $\gamma$ (x100 magnification). IFN- $\gamma$, interferon- $\gamma$.

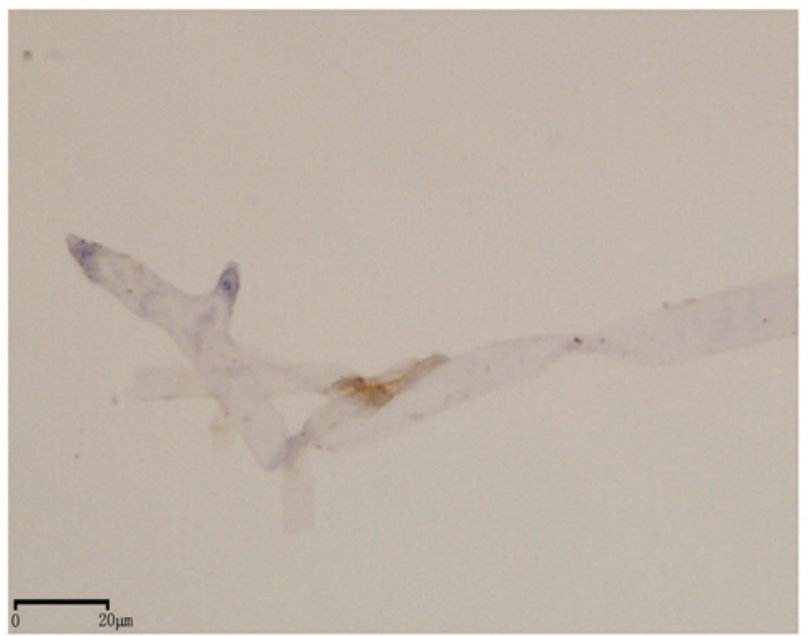

Figure 10. IFN- $\gamma$ immunostaining of Mucor spp cultures, which stained negative for IFN- $\gamma$ (x40 magnification). IFN- $\gamma$, interferon- $\gamma$.

and limited validation data for many fungal nucleic acid tests have hindered their general acceptance and broad application in clinical laboratories (16). The disadvantages of protein pattern recognition, such as matrix-assisted laser desorption ionization-time of flight mass spectrometry, include the requirement of culture (they are not amenable to direct sample testing), database supplementation (particularly for molds) and, occasionally, manual spectral analysis (19). The application of the antigen detection method in immunocompromised patients is limited. Although histopathology-based testing also possesses limitations, it has many advantages in clinical application, including the fact that pathologists are familiar with it, it is easy to perform, reagents are readily available, it provides rapid diagnosis and has relatively high accuracy. Therefore, at present, histopathological identification is the definitive method for diagnosing fungal sinusitis in clinics (20).

IFN- $\gamma$ is a protein dimer that has an essential role in the innate and adaptive phases of an immune response $(8,21,22)$. It is required for optimal activation of phagocytes, it collaborates 
in the generation of the protective antibody response and favors the development of a $\mathrm{T}$ helper 1 cell protective response $(7,8)$. In addition to polysaccharides, protein is also an important component of the fungal cell wall. However, prior to the present study, it was not established whether the fungal cell wall contained IFN- $\gamma$ antigen. In a pre-experiment on AIFRS animals, A. fumigatus in all infected cases exhibited brown staining when IFN- $\gamma$ antibody was applied. Therefore, it was hypothesized that IFN- $\gamma$ may be one of the components of the Aspergillus cell wall and IFN- $\gamma$ antibody may be used to identify the causative fungal types in cases of FRS.

The results of the present study demonstrated that all Aspergillus spp and Mucorales specimens exhibited positive staining with H\&E, PAS and GMS. GMS exhibited the strongest staining contrast between Aspergillus spp and Mucorales (data not shown). However, none of the tests accurately identified the fungal type, particularly for deformed and swollen fungi. Based on the principle of the specific antigen-antibody reaction, immunohistochemical staining with IFN- $\gamma$ antibody was markedly different between Aspergillus spp and Mucorales, 92.5\% Aspergillus spp stained positive, with diffuse brown staining on the cell wall, whereas Mucorales cell walls were all negatively stained, with the exception of one case. These results demonstrated that this may be a potentially useful, differential diagnostic test to distinguish between Aspergillus spp and Mucorales fungi.

In order to confirm that the IFN- $\gamma$ antigen was of fungal origin and not from the host tissue, the present study also performed immunocytochemistry on cultures of Aspergillus spp and Mucorales using IFN- $\gamma$ antibody. Again, IFN- $\gamma$ expression was observed in the cell walls of all Aspergillus spp, including A. fumigatus, A. flavus and A. terreus. By contrast, all Mucorales cell walls were negative. These results confirmed that IFN- $\gamma$ antibody may be used as a new supplementary test for the clinical diagnosis of FRS.

In order to verify that it was the IFN- $\gamma$ antibody, and not other substances present in the primary antibody, that had positively stained the Aspergillus spp, the present study applied a second IFN- $\gamma$ antibody produced by a different manufacturer to repeat the staining process and the observed results were identical, which further confirmed the reliability of this staining method for identifying fungal pathogens in FRS. In conclusion, immunohistochemical staining with IFN- $\gamma$ is a novel method for distinguishing between Aspergillus spp and Mucorales, and may be a beneficial supplementary test for current immunohistochemical methods in the diagnosis of FRS. IFN- $\gamma$ may be one of the antigen components of the cell wall of Aspergillus spp, however, this requires further studies.

\section{Acknowledgements}

The present study was supported in part by the Department of Pathology, Beijing Tongren Hospital (Beijing, China) and the National Natural Science Foundation of China (grant no. 81070769).

\section{References}

1. Chakrabarti A, Das A and Panda NK: Overview of fungal rhinosinusitis. Indian J Otolaryngol Head Neck Surg 56: 251-258, 2004.
2. Seo J, Kim HJ, Chung SK, Kim E, Lee H, Choi JW, Cha JH, Kim HJ and Kim ST: Cervicofacial tissue infarction in patients with acute invasive fungal sinusitis: Prevalence and characteristic MR imaging findings. Neuroradiology 55: 467-473, 2013.

3. Donovan ST, Thompson JW, Sandlund JT, Adderson EE, Pivnick EK and Harreld JH: Imaging of acute invasive fungal rhinosinusitis in a patient with gorlin syndrome and acute lymphocytic leukemia. Case Rep Otolaryngol 2013: 272314, 2013.

4. Kobayashi K, Kami M, Murashige N, Kishi Y, Fujisaki G and Mitamura T: Breakthrough zygomycosis during voriconazole treatment for invasive aspergillosis. Haematologica 89: ECR42, 2004.

5. Ma L, Xu R, Shi J, Zhou W, Xu G, Jiang G, Li G and Chen Z: Identification of fungi in fungal ball sinusitis: Comparison between MUC5B immunohistochemical and Grocott methenamine silver staining. Acta Otolaryngol 133: 1181-1187, 2013.

6. Piao YS, Zhang Y, Yang X, He CY and Liu HG: The use of MUC5B antibody in identifying the fungal type of fungal sinusitis. Hum Pathol 39: 650-656, 2008.

7. Kearney S, Delgado C and Lenz LL: Differential effects of type I and II interferons on myeloid cells and resistance to intracellular bacterial infections. Immunol Res 55: 187-200, 2013.

8. Gozalbo D, Maneu V and Gil ML: Role of IFN-gamma in immune responses to Candida albicans infections. Front Biosci (Landmark Ed) 19: 1279-1290, 2014.

9. Challa S, Uppin SG, Hanumanthu S, Panigrahi MK, Purohit AK, Sattaluri S, Borgohain R, Chava A, Vemu L and Jagarlapudi MM: Fungal rhinosinusitis: A clinicopathological study from South India. Eur Arch Otorhinolaryngol 267: 1239-1245, 2010.

10. Montone KT, Livolsi VA, Feldman MD, Palmer J, Chiu AG, Lanza DC, Kennedy DW, Loevner LA and Nachamkin I: Fungal rhinosinusitis: A retrospective microbiologic and pathologic review of 400 patients at a single university medical center. Int J Otolaryngol 2012: 684835, 2012.

11. Callejas CA and Douglas RG: Fungal rhinosinusitis: What every allergist should know. Clin Exp Allergy 43: 835-849, 2013.

12. Rupa V and Thomas M: Different types of fungal sinusitis occurring concurrently: Implications for therapy. Eur Arch Otorhinolaryngol 270: 603-608, 2013.

13. Chakrabarti A, Denning DW, Ferguson BJ, Ponikau J, Buzina W, Kita H, Marple B, Panda N, Vlaminck S, Kauffmann-Lacroix C, et al: Fungal rhinosinusitis: A categorization and definitional schema addressing current controversies. Laryngoscope 119: 1809-1818, 2009.

14. Aribandi M, McCoy VA and Bazan C III: Imaging features of invasive and noninvasive fungal sinusitis: A review. Radiographics 27: 1283-1296, 2007.

15. Thompson GR III and Patterson TF: Fungal disease of the nose and paranasal sinuses. J Allergy Clin Immunol 129: 321-326, 2012.

16. Wengenack NL and Binnicker MJ: Fungal molecular diagnostics. Clin Chest Med 30: 391-408, viii, 2009.

17. Ostrosky-Zeichner L: Invasive mycoses: Diagnostic challenges. Am J Med 125 (1 Suppl): S14-S24, 2012.

18. Griffin AT and Hanson KE: Update on fungal diagnostics. Curr Infect Dis Rep 16: 415, 2014.

19. Rosenvinge FS, Dzajic E, Knudsen E, Malig S, Andersen LB, Løvig A, Arendrup MC, Jensen TG, Gahrn-Hansen B and Kemp M: Performance of matrix-assisted laser desorption-time of flight mass spectrometry for identification of clinical yeast isolates. Mycoses 56: 229-235, 2013.

20. Michael RC, Michael JS, Ashbee RH and Mathews MS: Mycological profile of fungal sinusitis: An audit of specimens over a 7-year period in a tertiary care hospital in Tamil Nadu. Indian J Pathol Microbiol 51: 493-496, 2008.

21. Schroder K, Hertzog PJ, Ravasi T and Hume DA: Interferon-gamma: An overview of signals, mechanisms and functions. J Leukoc Biol 75: 163-189, 2004.

22. Toomer KH and Chen Z: Autoimmunity as a double agent in tumor killing and cancer promotion. Front Immunol 5: 116, 2014.

This work is licensed under a Creative Commons Attribution-NonCommercial-NoDerivatives 4.0 International (CC BY-NC-ND 4.0) License. 